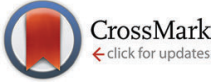

Cite this: J. Mater. Chem. C, 2016, 4, 4521

\section{Balancing structural distortions via competing $4 f$ and itinerant interactions: a case of polymorphism in magnetocaloric $\mathrm{HoCO}_{2} \dagger$}

\author{
Y. Mudryk, ${ }^{\star a}$ D. Paudyal, ${ }^{a}$ A. K. Pathak, ${ }^{a}$ V. K. Pecharsky ${ }^{\text {ab }}$ and \\ K. A. Gschneidner Jr. ${ }^{\text {ab }}$
}

Received 29th February 2016, Accepted 12th April 2016

DOI: $10.1039 / c 6 t c 00867 d$

www.rsc.org/MaterialsC

\begin{abstract}
The nature of multiple magnetostructural transformations in $\mathrm{HoCO}_{2}$ has been studied by employing magnetic and specific heat measurements, temperature and magnetic field dependent $\mathrm{X}$-ray powder diffraction, and first-principles calculations. Unexpected increase of magnetization observed below the spin-reorientation temperature $\left(T_{\mathrm{SR}}\right)$ suggests that the low-temperature transition involves a reduction of Co moment. First principles calculations confirm that the paramagnetic cubic to ferrimagnetic tetragonal transformation at $T_{\mathrm{C}}$ is assisted by itinerant electron metamagnetism, and that the reduction of Co moment in $\mathrm{HoCO}_{2}$ occurs in parallel with the ferrimagnetic tetragonal to the nearly ferromagnetic orthorhombic transformation at $T_{\mathrm{SR}}$ via the rearrangement of both $3 \mathrm{~d}$ states of Co and $5 \mathrm{~d}$ states of Ho. The ac magnetic susceptibility measurements show significant magnetic frustration below $T_{C}$. In contrast to earlier reports neither ac nor dc magnetic susceptibilities show anomalies in the paramagnetic region obeying the Curie-Weiss law.
\end{abstract}

\section{Introduction}

Among numerous compounds that undergo multiple phase transformations in response to varying temperature and/or magnetic field, an extended family of rare earth (R) - 3d transition metal (M) intermetallics continuously attracts research interest from both fundamental science and application perspectives. An in-depth understanding of the underlying science ultimately leads to practical applications, including permanent magnets, ${ }^{1,2}$ magnetostrictive devices and sensors, ${ }^{3}$ and magnetocaloric cooling $^{4,5}$ to name a few. Often mysterious magnetism in some of these materials arises from a complex interplay between $4 \mathrm{f}$ and $5 \mathrm{~d}$ electrons of lanthanides and $3 \mathrm{~d}$ electrons of transition metal counterparts. Importantly, as was recognized long ago when relatively pure rare earth metals became available in research quantities, ${ }^{6}$ the physical properties of $\mathrm{R}-\mathrm{M}$ compounds can be judiciously controlled by chemical modifications, thereby transforming them into an exciting playground for the investigation and better understanding of the ever elusive composition-structure-property relationships. As such, one may

\footnotetext{
${ }^{a}$ Division of Materials Science and Engineering, Ames Laboratory of US DOE, Iowa State University, Ames, Iowa, 50011-3020, USA. E-mail: slavkomk@ameslab.gov ${ }^{b}$ Department of Materials Science and Engineering, Iowa State University, Ames, Iowa, 50011-2300, USA

$\dagger$ Electronic supplementary information (ESI) available: Includes analysis of sample purity and basic magnetic characterization data. See DOI: 10.1039/c6tc00867d
}

expect that binary R-M systems have already been broadly and thoroughly studied, yet this remains far from being true even for some of the simplest rare earth-transition metal intermetallics. One example is an unusual interplay between structure and magnetism of the low temperature orthorhombic phase in the simple Laves phase compound - $\mathrm{HoCo}_{2}$ - that was reported long ago, ${ }^{7,8}$ but its origin has not been fully understood to this day.

The so-called Laves phases are one of the largest families known to exist among R-M compounds. Approximately 200 compounds with $\mathrm{RM}_{2}$ stoichiometry crystallize at room temperature in the three closely related C14 hexagonal $\mathrm{MgZn}_{2}$-type, C15 cubic $\mathrm{MgCu}_{2}$-type, and $\mathrm{C} 36$ hexagonal $\mathrm{MgNi}_{2}$-type structures. ${ }^{9}$ Many of $\mathrm{RM}_{2}$ compounds undergo structural distortions at low temperatures, which may or may not be coupled with magnetic ordering/reordering transformations. Depending upon the direction of the easy magnetization axis (EMA) that sets up at the magnetic transformation, cubic $\mathrm{RM}_{2}(\mathrm{M}=\mathrm{Co}$ or $\mathrm{Ni})$ compounds with magnetic rare earths $(\mathrm{R}=\mathrm{Pr}-\mathrm{Sm}, \mathrm{Gd}-\mathrm{Tm})$ may exhibit rhombohedral (for EMA $\langle 111\rangle$ ), tetragonal (EMA $\langle 100\rangle$ ), or orthorhombic (EMA $\langle 110\rangle$ ) distortions. ${ }^{8,10-12}$ When $\mathbf{M}=$ Co the distortions can be either first ( $\mathrm{R}=\mathrm{Dy}$, Ho, and $\mathrm{Er}$ ) or second order (for other lanthanides) transitions. ${ }^{13}$

Physical behaviours of $\mathrm{RCo}_{2}$ compounds are characterized by the intrinsic instability of Co magnetic moment and by a rich array of phenomena that such instability provides. ${ }^{14}$ Considering its neighbours in the periodic table (Fe and $\mathrm{Ni}$ ), $\mathrm{Fe}$ in $\mathrm{RFe}_{2}$ is always magnetic, while Ni remains non-magnetic in 
the corresponding isostructural $\mathrm{RNi}_{2}$ phases. ${ }^{14}$ Here, the nearly filled $3 \mathrm{~d}$ band $\left(3 \mathrm{~d}^{9}\right)$ of $\mathrm{Ni}$ is strongly hybridized with the $5 \mathrm{~d}$ band of the rare earth metal, which effectively fills the $3 \mathrm{~d}$ band quenching the magnetic moment of Ni. Magnetism of cobalt in $\mathrm{RCo}_{2}$ is, however, strongly dependent on a number of factors, either chemical (e.g. the nature of $\mathrm{R}$ element), or physical (applied pressure or magnetic field), or both; a similar instability is also present in $\mathrm{RMn}_{2}$ compounds, e.g. $\mathrm{TbMn}_{2},{ }^{15}$ due to halffilled $3 \mathrm{~d}$ shell of $\mathrm{Mn}$. At ambient pressure and zero magnetic field the net Co moment varies from $\sim 1 \mu_{\mathrm{B}}$ in $\mathrm{GdCo}_{2}$ to zero in $\mathrm{LuCo}_{2}$ and $\mathrm{YCo}_{2} \cdot{ }^{14}$ Application of very high magnetic field (up to $100 \mathrm{~T}$ ) induces metamagnetism in non-magnetic $\mathrm{YCo}_{2}$ and $\mathrm{LuCo}_{2} \cdot{ }^{16}$ Crystalline-electric fields $(\mathrm{CEF})^{17,18}$ and magnetostructural coupling are strongly affecting both the magnetism and crystal structure of $\mathrm{RCo}_{2}$ compounds.

$\mathrm{NdCo}_{2}$ and $\mathrm{HoCo}_{2}$ occupy a special place among all $\mathrm{RCo}_{2}$ compounds because they undergo not one but two crystallographic transitions: first is the high temperature tetragonal distortion of the cubic structure at the magnetic ordering temperature, second is the low temperature orthorhombic deformation of the tetragonal structure at the spin-reorientation transition. ${ }^{7,8,19} \mathrm{HoCo}_{2}$, however, is the only member of the series where the cubic-tetragonal transition is first-order in nature while the tetragonal-orthorhombic one is assumed to be second-order ${ }^{11,13}$ (both transitions in $\mathrm{NdCo}_{2}$ are second-order), although early measurements of the heat capacity of $\mathrm{HoCo}_{2}$ suggested that both transitions may be first-order. ${ }^{20,21}$

$\mathrm{HoCo}_{2}$ orders ferrimagnetically at $T_{\mathrm{C}}=76 \mathrm{~K}$ during cooling with EMA $\langle 100\rangle$ and exhibits a narrow $(\sim 2 \mathrm{~K})$ thermal hysteresis around $T_{\mathrm{C}} \cdot{ }^{20}$ The EMA changes to $\langle 110\rangle$ at the spin-reorientation transition that, depending on the sample, occurs at $T_{\mathrm{SR}}=$ 14-16 K. ${ }^{20,22}$ Both magnetic transitions are coupled with structural transformations, which are basically distortions of the original C15 cubic structure along the directions of EMA. Specific heat measurements confirm the presence of two distinct transitions in $\mathrm{HoCo}_{2}{ }^{21,23}$ Two clearly defined peaks were observed in ac susceptibility, $\chi_{\mathrm{ac}}(T)$, of $\mathrm{HoCo}_{2}$ at $T_{\mathrm{C}}$ and at $T_{\mathrm{SR}}$, and two additional minor anomalies (at $\sim 40 \mathrm{~K}$ and near $T_{\mathrm{SR}}$ ) are present as well. ${ }^{24}$ Bonilla et al. ${ }^{25}$ observed two major anomalies at $T_{\mathrm{C}}$ and $T_{\mathrm{SR}}$ plus a weak anomaly in the paramagnetic region at $126 \mathrm{~K}$. Apparently, the $\chi_{\mathrm{ac}}(T)$ data are sample dependent even though the singlephase composition of $\mathrm{HoCo}_{2}$ alloys is claimed in nearly all published studies.

Spontaneous magnetization of $\mathrm{HoCo}_{2}$ crystal along $\langle 110\rangle$ EMA is $7.7 \mu_{\mathrm{B}}$ per f.u. at $4.2 \mathrm{~K}^{20}$ The holmium magnetization calculated in the same study is $9.3 \mu_{\mathrm{B}}$ per Ho leading to $-0.8 \mu_{\mathrm{B}}$ per Co moment. ${ }^{20}$ Neutron diffraction and magnetization measurements performed on a $\mathrm{HoCo}_{2}$ sample with a higher $T_{\mathrm{C}}=87 \mathrm{~K}$ (indicating possible compositional deviation from the ideal stoichiometry) led to similar values of $\mu_{\mathrm{Ho}}=9.5 \mu_{\mathrm{B}}$ and $\mu_{\mathrm{Co}}=-1.0 \mu_{\mathrm{B}} \cdot{ }^{26}{ }^{59} \mathrm{Co}$ nuclear magnetic resonance study of $\mathrm{HoCo}_{2}$ gives a slightly lower cobalt moment of $0.84 \mu_{\mathrm{B}}$ per Co. ${ }^{27}$

It is believed that the cobalt magnetization is exchange induced by the lanthanides, ${ }^{14}$ and that their molecular field may lead to a non-zero value of Co moment in the paramagnetic state. In addition, spin fluctuations were observed and are strongly affecting electronic transport in the paramagnetic region above $T_{\mathrm{C}}$ in all $\mathrm{RCo}_{2}$ compounds, including $\mathrm{HoCo}_{2} \cdot{ }^{28} \mathrm{X}$-ray circular magnetic dichroism (XMCD) study of $\mathrm{HoCo}_{2}$ found that XMCD signals of both Ho and Co do not vanish above $T_{\mathrm{C}}$, confirming the existence of spin fluctuations. ${ }^{29}$ "Enhanced" Pauli susceptibility of Co sublattice, essentially temperature independent, was detected by polarized neutron diffraction in the paramagnetic region of $\mathrm{HoCo}_{2} \cdot{ }^{30}$ The higher value of Co susceptibility (as compared to nonmagnetic $\mathrm{YCo}_{2}$ ) was explained by the presence of high molecular field of $\mathrm{R}$ atoms acting upon the Co atoms. At the same time the temperature evolution of the Co magnetic moment in $\mathrm{ErCo}_{2}$ single crystal measured using polarized neutron diffraction was found to be temperature dependent. ${ }^{31}$

It is worth to note that the presence of the Co net magnetic moment in the paramagnetic state is counterintuitive, but if one assumes such possibility, then the Co moment must be oriented antiparallel to the rare earth moment. ${ }^{14}$ The antiparallel alignment of $\mathrm{R}$ and Co magnetic sublattices in the paramagnetic phase was recently attributed to the occurrence of short-range correlations at $T>T_{\mathrm{C}} \cdot{ }^{25,31-33}$ Observed in several $\mathrm{RCo}_{2}$ compounds, including $\mathrm{HoCo}_{2}$, this unusual magnetism was dubbed "parimagnetism". Parimagnetism was explained in terms of Griffiths phase-like behaviour. ${ }^{25}$ In fact, the existence of two different paramagnetic phases, one immediately above $T_{\mathrm{C}}$ and another one at $T \gg T_{\mathrm{C}}$ was reported in ref. 25 . Recent theoretical calculations indicate that the interstitial impurities and antisite substitutions play a role in the formation of shortrange ferromagnetic Co clusters in $\mathrm{RCo}_{2} \cdot{ }^{34}$ With application of magnetic field these clustered Co atoms develop spin polarization leading to the observed parimagnetism.

Sharp changes in physical properties occur in $\mathrm{HoCo}_{2}$ at the first-order magnetostructural phase transition at $T_{\mathrm{C}} \cdot^{28,35-38}$ Latent heat and magnetovolume effect ${ }^{39}$ confirm that the first-order nature of this transition is related to the changes in the crystal lattice. Magnetic, electronic transport, and thermal properties of $\mathrm{HoCo}_{2}$ show that the $T_{\mathrm{C}}$ is susceptible to changes of magnetic field and applied pressure $(P):{ }^{23,36-39} T_{\mathrm{C}}$ increases with the increasing magnetic field, while hydrostatic pressure reduces $T_{\mathrm{C}}$ because ferrimagnetic $\mathrm{HoCo}_{2}$ is the high volume phase. ${ }^{14,35,40}$ At $p \sim 3 \mathrm{GPa}$ the transition becomes second-order and no change of $T_{\mathrm{C}}$ is observed at higher pressures. ${ }^{36,38}$ The first-order nature of the transition in $\mathrm{HoCo}_{2}$ is preserved at least up to $5 \mathrm{~T}$. $^{39}$

The sensitivity of the first-order paramagnetic-ferrimagnetic transition to the applied magnetic field leads to several potentially useful effects in $\mathrm{HoCo}_{2}$ near $T_{\mathrm{C}}$, such as large magnetostriction, magnetoresistance, and giant magnetocaloric effect (GMCE). Spontaneous volume expansion $\Delta V / V$ of $\sim 4 \times 10^{-3}$ at $T_{\mathrm{C}}$ was reported. $^{35}$ Magnetostriction constants, $\lambda_{100}$ and $\lambda_{111}$, are $-2.2 \times 10^{-3}$ and $-0.5 \times 10^{-3}$, respectively. ${ }^{10}$ Large magnetoresistance $(-50 \%)$ was demonstrated above $T_{\mathrm{C}}$ in $\mathrm{HoCo}_{2}$ single crystals oriented along [100], [111], [110] crystallographic directions. ${ }^{41}$ Large values of magnetic entropy $\left(\Delta S_{\mathrm{M}}\right)$ and adiabatic temperature $\left(\Delta T_{\text {ad. }}\right)$ change were obtained for $\mathrm{HoCo}_{2}$ : for a magnetic field change from 0 to $8 \mathrm{~T}, \Delta S_{\mathrm{M}}=-8 \mathrm{~J} \mathrm{~mol}^{-1} \mathrm{~K}^{-1}\left(-28.3 \mathrm{~J} \mathrm{~kg}^{-1} \mathrm{~K}^{-1}\right)$ and $\Delta T_{\mathrm{ad} .}=10 \mathrm{~K}^{23}$ Direct measurements of magnetocaloric effect in $\mathrm{HoCo}_{2}$ revealed $\Delta T_{\mathrm{ad} .}=5.1 \mathrm{~K}$ for $6 \mathrm{~T}$ magnetic field 
change at $82 \mathrm{~K} .{ }^{42}$ Barocaloric effect was estimated theoretically and is expected to be similar in magnitude to the magnetocaloric one. ${ }^{43}$

While the physical behaviours of $\mathrm{HoCo}_{2}$ were extensively studied near the magnetic ordering transition, the region below $T_{\mathrm{C}}$ remains less investigated. In particular, the origin of the structural transformation at $T_{\mathrm{SR}}$, which does not fit within the IEM model, and the unusual behaviour of lattice parameters and magnetization for the orthorhombic $\mathrm{HoCo}_{2}$ have emerged as interesting fundamental problems. Here, we report an in-depth investigation and analysis of these problems via both the experimental investigation of $\mathrm{HoCo}_{2}$ prepared using highpurity metals and the first-principles calculations employing experimentally determined near ground state crystallographic data.

\section{Experimental techniques}

Polycrystalline $\mathrm{HoCO}_{2}$ alloy $(\sim 5 \mathrm{~g})$ was arc-melted from the elements in an Ar atmosphere using high purity Ho metal prepared by the Materials Preparation Center at the Ames Laboratory of the U.S. Department of Energy; ${ }^{44}$ cobalt metal (Puratronic, 99.9+\% purity) was purchased from Alfa Aesar. The Ho was at least 99.7 at\% pure with respect to all elements in the Periodic Table. Synthesis of the $\mathrm{HoCo}_{2}$ compound using the stoichiometric quantities of Ho and Co resulted in the alloy containing a minor amount of the $\mathrm{HoCo}_{3}$ phase (sample I). The presence of the $\mathrm{HoCo}_{3}$ impurity could not influence the low-temperature X-ray diffraction (XRD) investigation of the material's crystal structure but was detrimental to the physical property measurements. Therefore, an additional amount of Ho (3 wt $\%$ ) was added during the second synthesis in order to compensate for the Ho loss from evaporation and to prevent the formation of the $\mathrm{HoCo}_{3}$ impurity phase. The alloy was melted and thoroughly mixed, then allowed to solidify, turned over, and re-melted again to ensure homogeneity; the button broke into several pieces during cooling after the second melting. After melting the alloy was sealed in a helium-filled quartz ampoule, heat-treated for 5 days at $900{ }^{\circ} \mathrm{C}$, and quenched in water. The room temperature X-ray powder diffraction pattern indicated that the material is the single phase $\mathrm{HoCo}_{2}$ (sample II) crystallizing in the $\mathrm{MgCu}_{2}$-type structure with lattice parameter $a=$ 7.1750(1) ̊̊ (Fig. S1, ESI $\dagger$ ). Sample II phase purity was confirmed using backscatter scanning electron microscopy (Fig. S2, ESI $\dagger$ ) and this sample was used in the physical property measurements.

The temperature and magnetic field dependencies of $\mathrm{dc}$ magnetization and ac magnetic susceptibility were studied using Quantum Design Superconducting Quantum Interference Device (SQUID) magnetometer, model MPMS XL-7. The heat capacity was measured first using a semi-adiabatic heat pulse calorimeter ${ }^{45}$ in magnetic fields from 0 to $100 \mathrm{kOe}$; later the detailed study of the low-temperature heat capacity was performed using both ${ }^{4} \mathrm{He}$ and ${ }^{3} \mathrm{He}$ probes of the Quantum Design Physical Property Measurement System (PPMS).

The temperature and magnetic field dependent X-ray powder diffraction measurements were performed using the
$\mathrm{HoCo}_{2}$ powder (sample I, particle size $<25 \mu \mathrm{m}$, mixed with GE varnish) at temperatures ranging from 5 to $300 \mathrm{~K}$ and in magnetic fields between 0 and $40 \mathrm{kOe}$. The data were collected on a Rigaku TTRAX system with a rotating anode generating Mo $\mathrm{K} \alpha$ radiation. The range of measured $2 \theta$ angles was from 8 to $63 \mathrm{deg}$ with a $0.01 \mathrm{deg}$ step. Further details about sample preparation and experimental setup can be found in ref. 46 .

\section{Experimental results}

\section{Magnetic properties}

The dc magnetization, measured as a function of temperature, $M(T)$, during warming of zero-field-cooled (ZFC) sample, in-field cooling (FC), and warming of in-field cooled sample (FCW) regimes in a $H=100$ Oe applied magnetic field, shows a sharp first-order type magnetic ordering transition at $T_{\mathrm{C}}=76 \mathrm{~K}$ (Fig. 1). The spin-reorientation transition, manifested as a local maximum on the low-field $M(T)$ curves and as a step on the high-field $M(T)$ curves (see Fig. 2), occurs at $T_{\mathrm{SR}}=14 \mathrm{~K}$. Contrary to the earlier report $^{20}$ the transition at $T_{\mathrm{C}}$ is non-hysteretic despite its distinct first-order nature. At the same time, substantial thermomagnetic irreversibility occurs at $T_{\mathrm{SR}}<T<T_{\mathrm{C}}$ between all three (ZFC, FC, and FCW) measurements. Below $T_{\mathrm{SR}}$ the FC and FCW curves coincide, while the ZFC magnetization is lower, most likely due to domain wall pinning ("freezing") effect. The irreversibility at $T_{\mathrm{SR}}<T<T_{\mathrm{C}}$, however, is harder to explain by a trivial domain effect as FC and FCW dependencies significantly separate from each other above $T_{\mathrm{SR}}$; furthermore, there is an apparent crossover between FC and FCW curves in the region of the spin-reorientation transition. Such separation usually indicates sizeable magnetic frustrations in the system. Moreover, an additional magnetic anomaly is seen above $T_{\mathrm{SR}}$ indicating that the thermal evolution of magnetic properties below $T_{\mathrm{C}}$ in $\mathrm{HoCo}_{2}$ involves more than a single-step reorientation of EMA from $\langle 100\rangle$ to $\langle 110\rangle$ direction at $T_{\mathrm{SR}}$.

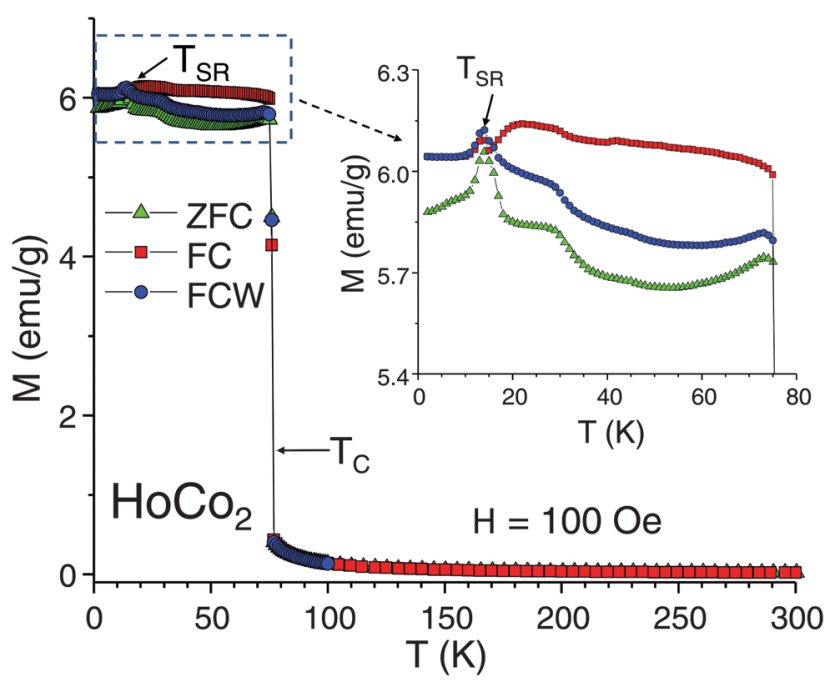

Fig. 1 The low-field dc magnetization of $\mathrm{HoCO}_{2}$ measured as a function of temperature at $H=100 \mathrm{Oe}$. The inset magnifies the temperature range below $76 \mathrm{~K}$ where $\mathrm{HoCO}_{2}$ is magnetically ordered. 

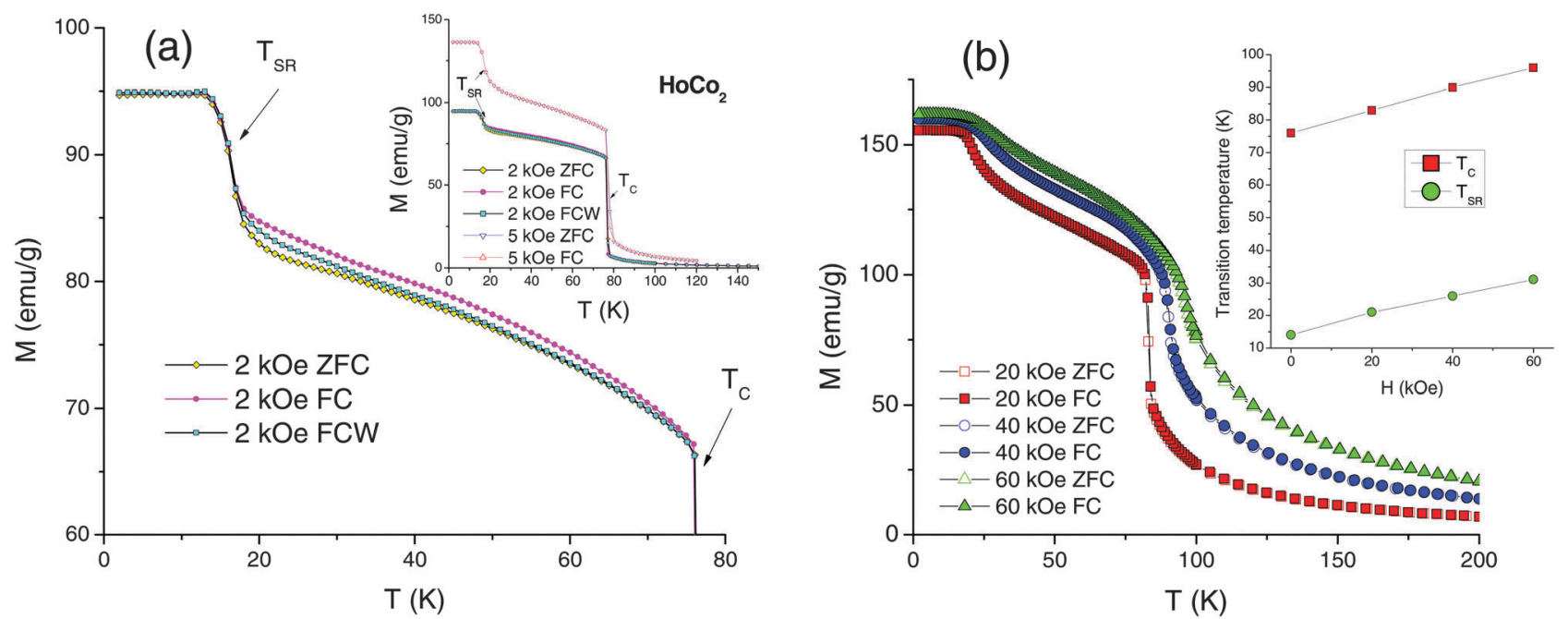

Fig. 2 The ZFC, FC, and FCW high-field dc magnetization of $\mathrm{HoCO}_{2}$ measured as a function of temperature: (a) data collected at $\mathrm{H}=2 \mathrm{kOe}$ (comparison of 2 and $5 \mathrm{kOe}$ data is shown in the inset); (b) data collected at 20,40, and $60 \mathrm{kOe}$. The inset shows the magnetic field dependence of the magnetic transition temperatures.

Reciprocal magnetic susceptibility of $\mathrm{HoCo}_{2}$ measured in 100 Oe magnetic field shows that in the paramagnetic region $\mathrm{HoCo}_{2}$ follows the Curie-Weiss law (Fig. S3, ESI $\dagger$ ). Minor but interesting differences between as cast and annealed samples are observed, even though the X-ray patterns of both samples are practically identical and indicate a single phase material. For the as cast sample, a weak negative deviation from linearity is seen in the $T_{\mathrm{C}}<T<150 \mathrm{~K}$ temperature range (Fig. S3a, ESI $\dagger$ ), which agrees with the earlier reported magnetic anomaly. ${ }^{25}$ Heat-treatment completely removes this anomaly (Fig. S3b, ESI $\dagger$ ). The important conclusion here is that the short-range order effects and/or the parimagnetism reported in ref. 25, 29 and 30 may not be intrinsic to $\mathrm{HoCo}_{2}$; they are indeed sample dependent, and the phase purity of materials plays a significant role.

The effective moment calculated from the Curie-Weiss law is $10.51 \mu_{\mathrm{B}}$ per f.u. - in good agreement with the calculated $g_{J}[J(J+1)]^{1 / 2}=10.61 \mu_{\mathrm{B}}$ per $\mathrm{Ho}^{3+}$, and the Weiss temperature is $64 \mathrm{~K}$, which is slightly lower than $T_{\mathrm{C}}=76 \mathrm{~K}$.

The $M(T)$ measurements preformed in magnetic fields 2,5 , 20, 40, and 60 kOe are shown in Fig. 2. Weak magnetic irreversibility between $T_{\mathrm{SR}}$ and $T_{\mathrm{C}}$ persists in 2 kOe applied field (Fig. 2a), but the magnetization becomes reversible at $H \geq 5 \mathrm{kOe}$ (inset of Fig. 2a and b). The transition at $T_{\mathrm{SR}}$ is clearly seen in high magnetic fields, including 60 kOe data, but minor $M(T)$ anomalies observed in 100 Oe field (Fig. 1) are suppressed at $2 \mathrm{kOe}$ and above. Magnetization jump at $T_{\mathrm{SR}}$ is most pronounced in $1 \mathrm{kOe} \leq H \leq$ 5 kOe magnetic fields. Both $T_{\mathrm{SR}}$ and $T_{\mathrm{C}}$ (defined as minima of $\mathrm{d} M / \mathrm{d} T)$ increase with the application of magnetic field with $\mathrm{d} T_{\mathrm{C}} / \mathrm{d} H=0.33 \mathrm{~K} \mathrm{kOe}^{-1}$ being slightly higher than $\mathrm{d} T_{\mathrm{SR}} / \mathrm{d} H=$ $0.28 \mathrm{~K} \mathrm{kOe}^{-1}$ (inset of Fig. 2b). The sharp first-order character of the magnetic ordering transition is notably suppressed by the magnetic field - the paramagnetic-ferrimagnetic (PM-FIM) transition at 60 kOe visually appears as a typical second-order phenomenon.
The magnetization as a function of applied magnetic field was measured at 5, 50, 80, and $100 \mathrm{~K}$, see Fig. S4 (ESI $\dagger$ ). No magnetic hysteresis/coercivity was observed in $5 \mathrm{~K}$ data indicating distinctively soft nature of polycrystalline $\mathrm{HoCo}_{2}$ at this temperature. The saturated magnetic moment obtained from extrapolation of $M(H)$ data in $M v s .1 / H$ plot is equal to $8.4 \mu_{\mathrm{B}}$ per f.u. Assuming that the holmium moment is equal to $g_{J}=$ $10 \mu_{\mathrm{B}}$ per $\mathrm{Ho}^{3+}$ and considering antiparallel alignment of Ho and Co atoms, the Co moment is $0.8 \mu_{\mathrm{B}}$ per Co, which basically agrees with earlier reports. ${ }^{20,26,27}$ It is noted that all of the neutron diffraction studies reported Ho moment lower than $10 \mu_{\mathrm{B}}$, generally $\sim 9.5 \mu_{\mathrm{B}}$ (the reduction of the Ho moment is probably due to CEF splitting), which means that the true Co moment in our $\mathrm{HoCo}_{2}$ at $5 \mathrm{~K}$ may be lower, i.e. $\sim 0.5-0.6 \mu_{\mathrm{B}}$ (this will be further discussed in the theory section). The $M(H)$ isotherms at 50 and $100 \mathrm{~K}$ are characteristic for the ferrimagnetic and paramagnetic states, respectively. The observed reversible metamagnetic behaviour and magnetic hysteresis at $80 \mathrm{~K}$ are typical for the magnetic-field-induced PM-FIM magnetostructural transformation due to Co IEM behaviour which is set up by the emerging internal magnetic field of the Ho sublattice.

A sudden increase of magnetization observed during cooling of $\mathrm{HoCo}_{2}$ in moderate magnetic fields at $T_{\mathrm{SR}}$ (Fig. 2a) is by itself an interesting yet rarely discussed phenomenon. It has been observed not only in $\mathrm{HoCo}_{2}$ but also in mixed rare-earth alloys like $\mathrm{Ho}_{0.7} \mathrm{Y}_{0.3} \mathrm{Co}_{2},{ }^{47}$ and even though the size of this anomaly decreases with increasing magnetic field strength it is present in 60 kOe field as well (Fig. 2b). The possible explanations for such an increase are (1) a transition from a non-collinear (canted) magnetic structure of Ho sublattice to a collinear one at $T_{\mathrm{SR}}$; (2) strong texture present in polycrystalline $\mathrm{HoCo}_{2}$; (3) a decrease of Co moment caused by the changes in the electronic structure due to the structural distortion. The first hypothesis is unlikely because it assumes that Ho and Co moments are not antiparallel below $T_{\mathrm{C}}$ (canted magnetic structure) meaning that 
there is a ferromagnetic component in the $\mathrm{HoCo}_{2}$. The second scenario is based on the study of the $\mathrm{HoCo}_{2}$ single crystal by Gignoux et al., ${ }^{20}$ which shows that along the [110] direction the magnetization saturates quickly at $14.4 \mathrm{~K}$ (below $T_{\mathrm{SR}}$ ) but requires strong magnetic field on the order of $\sim 50 \mathrm{kOe}$ for the saturation at $25 \mathrm{~K}$ (above $T_{\mathrm{SR}}$ ). The main caveat here is that there is no reason why the cubic $\mathrm{HoCo}_{2}$ should develop strong texture along [110] direction during solidification. Moreover, the results of the $M(T)$ measurements performed using the $\mathrm{HoCo}_{2}$ powder (not shown) are similar to the results of the bulk sample measurements shown in the Fig. 2.

The third possibility suggests that Co moment changes as a result of cubic-tetragonal distortion in response to the change of lattice parameters and symmetry. The X-ray powder diffraction data (discussed in detail below) indicate (1) shallow but steady decrease of the unit-cell volume below $T_{\mathrm{SR}}$ and (2) a tendency toward restoring the original cubic structure as the "c/a" ratio moves closer to 1 below $T_{\mathrm{SR}}$, see Fig. 8c, below. According to the pressure-dependent study by Burzo et al., ${ }^{47}$ volume contraction in the magnetically ordered state leads to a smaller Co moment, which agrees with the fact that the increase in the net magnetization coincides with the decrease of the unitcell volume at $T_{\mathrm{SR}}$. The change of the $c / a$ ratio can also affect the magnetic moment. ${ }^{47}$ Our own theoretical investigation presented below clearly confirms that the structural transformation is the primary reason for the reduction of Co moment below $T_{\mathrm{SR}}$.

The AC susceptibility was employed to study magnetic anomalies reported above the magnetic ordering temperature (ref. 25) as well as those observed below the $T_{\mathrm{C}}$ (seen in the low-field $M(T)$ data of Fig. 1, and in the $\chi_{\mathrm{AC}}(T)$ data of ref. 24,25). The $\chi_{\mathrm{AC}}(T)$ measurements were performed at AC field frequencies $f=1,100$, and $1000 \mathrm{~Hz}$ (Fig. 3). The data show that: (1) there is a strong frequency dependence of the AC magnetic susceptibility in the magnetically ordered $\mathrm{HoCo}_{2}$, especially in the imaginary component; (2) there are no magnetic anomalies in the $1 / \chi_{\mathrm{AC}}{ }^{\prime} v s$. $T$ plot above $T_{\mathrm{C}} ;(3)$ regions where $\mathrm{d} \chi^{\prime} / \mathrm{d} f \neq 0$ alternate with regions where there is no frequency dependence. The strongest frequency dependence, which is commonly associated with sizeable magnetic frustrations and spin-glass-like behaviour, occurs between $\sim 25$ and $\sim 65 \mathrm{~K}$. Unlike $\chi^{\prime \prime}$, whose dependence upon $f$ extends to all temperatures below $T_{\mathrm{C}}$, the $\mathrm{d} \chi^{\prime} / \mathrm{d} f=0$ near the magnetostructural transitions $\left(T_{\mathrm{SR}}\right.$ and $\left.T_{\mathrm{C}}\right)$. One can safely rule out the presence of spin-glass state in $\mathrm{HoCo}_{2}$ because the long-range magnetic order is well established below $T_{\mathrm{C}}$. One possible explanation is that because the magnetic structure with $\langle 100\rangle$ EMA is not the stable ground state, energetically close magnetic structures (with EMA $\langle 100\rangle$ and EMA $\langle 110\rangle$ ) are competing with each other between $T_{\mathrm{C}}$ and $T_{\mathrm{SR}}$ until the ground state structure with EMA $\langle 110\rangle$ is established at $T_{\mathrm{SR}}$. This means that the magnetic frustrations in $\mathrm{HoCo}_{2}$ originate from the random magnetic anisotropy effect most likely related to the single-ion random anisotropy ${ }^{48}$ of Ho moments. This effect is similar to frustrations caused by a competition between fourthorder anisotropy terms of $\mathrm{Er}$ and Dy in $\mathrm{Er}_{0.75} \mathrm{Dy}_{0.25} \mathrm{Al}_{2}{ }^{49}$ and different from the one occurring in the amorphous random anisotropy magnets, such as NdGdFe thin films. ${ }^{50}$ The interesting question here is whether this frustration is related to the theoretically established competition between $4 \mathrm{f}$ and IEM magnetism (discussed below in the Theory section).

Another interesting problem is the sudden loss of the frequency dependence in $\chi^{\prime}(T)$ between the $T_{\mathrm{SR}}$ and a local susceptibility minimum at $\sim 25 \mathrm{~K}$. The energy losses reflected in $\chi^{\prime \prime}(T)$ below $T_{\mathrm{SR}}$, especially for the high-frequency data, are associated with the domain-wall-pinning effects, which essentially disappear above $T_{\mathrm{SR}}$. It would be interesting to compare AC susceptibility behaviour of $\mathrm{HoCo}_{2}$ with that of other $\mathrm{RCo}_{2}$ systems (namely $\mathrm{ErCo}_{2}$ and $\mathrm{DyCo}_{2}$ ) that do not have the second structural transition at $T_{\mathrm{SR}}$, but have a first order transition at $T_{\mathrm{C}}$.

\section{Heat capacity}

The heat-capacity investigation was performed in the three separate experiments. At first, the heat capacity of a large bulk
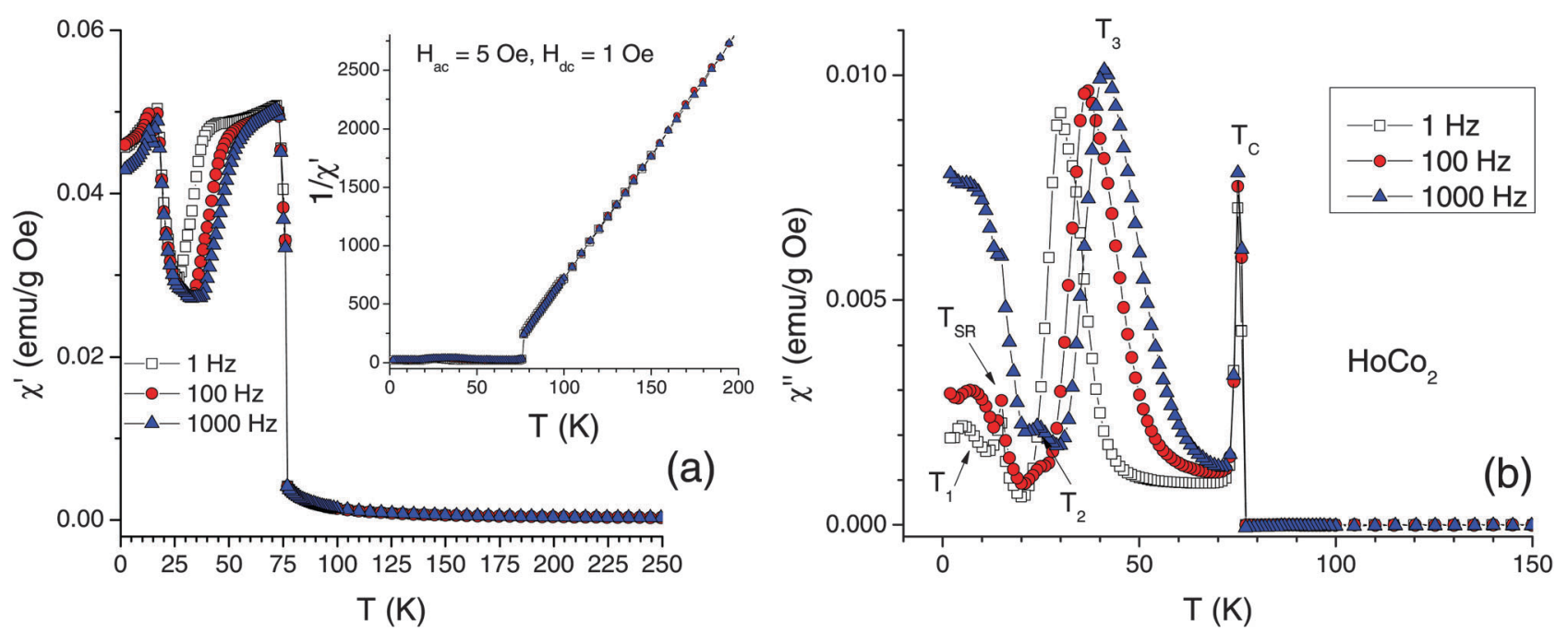

Fig. 3 The $\mathrm{AC}$ magnetic susceptibility of $\mathrm{HoCO}_{2}$ measured as a function of temperature: (a) the real part (the inset shows the reciprocal susceptibility); (b) the imaginary part. 
polycrystalline sample ( $\sim 1 \mathrm{~g}$ ) was measured from $2 \mathrm{~K}$ to room temperature in magnetic fields ranging from 0 to $100 \mathrm{kOe}$ using a semi-adiabatic calorimeter. The results of these measurements agree very well with those previously reported in ref. 23 and, therefore, are not shown here. In agreement with ref. 23 a sharp first-order type peak is observed in zero-field data at $T_{\mathrm{C}}$, and a second, much weaker anomaly occurs at $T_{\mathrm{SR}}$. No other anomalies are observed in this temperature range. Application of magnetic field progressively decreases the sharpness of the peak at the first-order transition. Compared to some other first-order transitions that remain almost as sharp at $100 \mathrm{kOe}$ as at $0 \mathrm{kOe}$ (e.g. some $\mathrm{Gd}_{5} \mathrm{Ge}_{4}$-based alloys, see Fig. 3 in ref. 51 and Fig. 9 in ref. 52), the magnetic ordering transition in $\mathrm{HoCo}_{2}$ gradually changes from clearly discontinuous at $H=0$ kOe to nearly continuous in high magnetic fields. According to a previous calorimetric investigation ${ }^{39}$ the transition at $T_{\mathrm{C}}$ in $\mathrm{HoCo}_{2}$ is firstorder at $50 \mathrm{kOe}$, but higher magnetic fields were not studied. It is difficult to say unequivocally if the certain order (first or second) of the transition can be assigned to $\mathrm{HoCo}_{2}$ in a 100 kOe applied magnetic field. However, taking into account the influence of the magnetic field on the structural transition (see below), particularly the magnetic-field-induced suppression of the discontinuous volume change, we believe that at sufficiently high magnetic fields the nature of the order of the transformation in $\mathrm{HoCo}_{2}$ changes from first order to second order.

The second series of measurements was performed in a Quantum Design PPMS using the standard heat-capacity option. The measurements were focused on the heat-capacity behaviour in the vicinity of $T_{\mathrm{SR}}$, because it has not been studied before in any detail. The zero-field heat capacity data show the $\lambda$-type specific heat anomaly at $16 \mathrm{~K}$ (Fig. 4), which generally agrees with the $T_{\mathrm{SR}}=14 \mathrm{~K}$ determined from magnetization measurements (Fig. 1). The application of magnetic field has little effect at $H=5 \mathrm{kOe}$, but at $H \geq 10$ kOe the transition becomes significantly broader and moves towards higher temperatures. Considering all of the above, the transformation at $T_{\mathrm{SR}}$ appears to be a second-order one.

In addition to the anomaly at $T_{\mathrm{SR}}$, Fig. 4 shows a weak but clear upturn of $C_{\mathrm{p}}$ below $\sim 4 \mathrm{~K}$, which arises due to a strong hyperfine field at the nuclei created by orbital and spin angular momenta of the lanthanide ions. ${ }^{53}$ Earlier studies reported that $\mathrm{Pr}, \mathrm{Tb}$ and Ho metals show enhanced heat capacity at $T<2 \mathrm{~K}^{54}$ Because a study of $C_{\mathrm{p}}$ at $T<2 \mathrm{~K}$ has not been performed for $\mathrm{HoCo}_{2}, C_{\mathrm{p}}$ was explored down to $0.4 \mathrm{~K}$ in magnetic fields up to 140 kOe using PPMS Quantum Design ${ }^{3}$ He option. Fig. 5 shows that $C_{\mathrm{p}}$ increases rapidly below $2 \mathrm{~K}$ and reaches $\sim 7370 \mathrm{~mJ} \mathrm{~mol}^{-1} \mathrm{~K}^{-1}$ at $T=0.4 \mathrm{~K}$ and $H=0 \mathrm{kOe}$, which is similar to pure Ho metal, $7000 \mathrm{~mJ} \mathrm{~mol}{ }^{-1} \mathrm{~K}^{-1} \cdot{ }^{54}$ For $C_{\mathrm{p}}$ measurement in $140 \mathrm{kOe}$ field our lowest temperature was $0.8 \mathrm{~K}$; the starting temperatures for measurements in intermediate magnetic fields were between 0.4 and $0.8 \mathrm{~K}$. For the sake of consistency, $C_{\mathrm{p}}$ was fitted for the $0.8 \leq T \leq 2 \mathrm{~K}$ region as:

$$
C_{\mathrm{p}}=\gamma T+\beta T^{3}+C_{\mathrm{N}} T^{-2}
$$

where the standard electronic $(\gamma T)$ and lattice $\left(\beta T^{3}\right)$ contributions ${ }^{53}$ are supplemented by the nuclear specific heat $\left(C_{\mathrm{N}} T^{-2}\right)$ term.

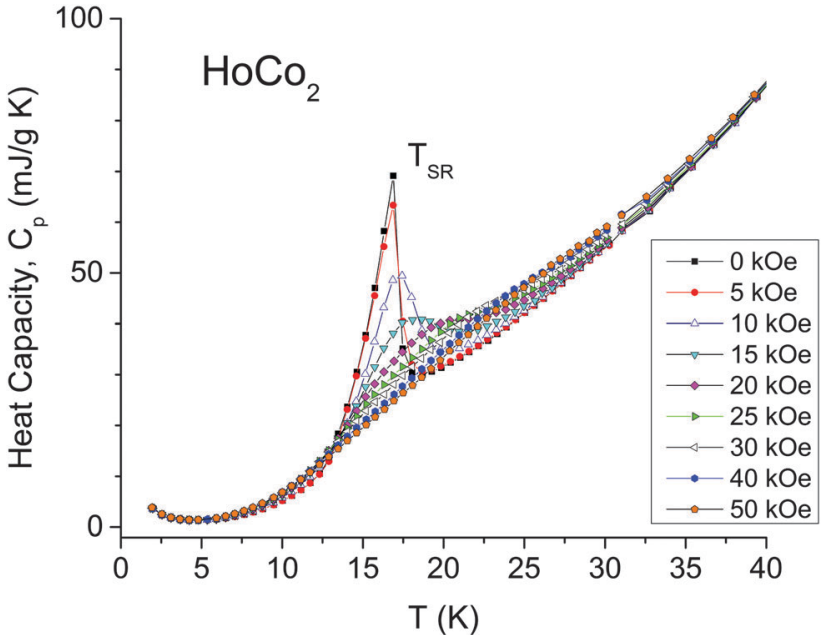

Fig. 4 Temperature dependence of the heat capacity of $\mathrm{HoCO}_{2}$ in the vicinity of the spin-reorientation transition measured in magnetic fields ranging from 0 to $50 \mathrm{kOe}$.

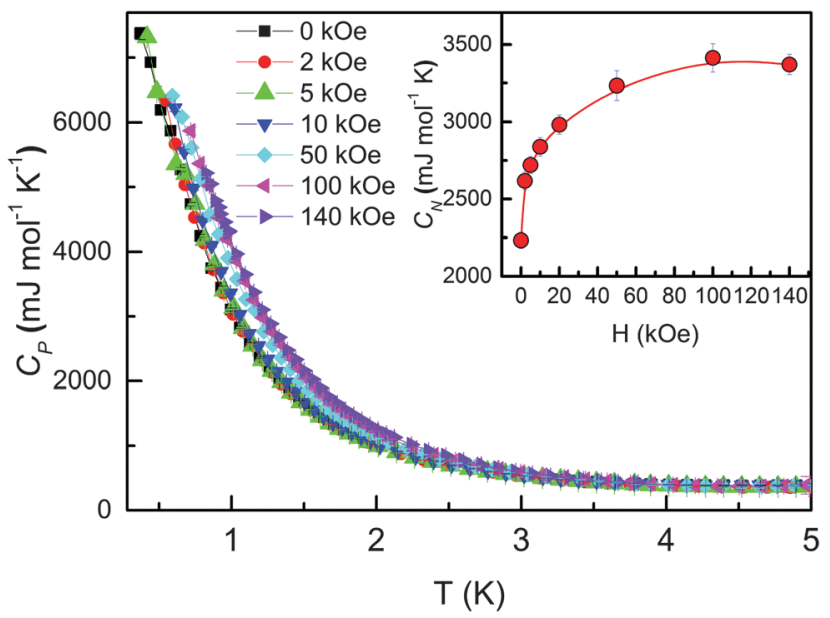

Fig. 5 The heat capacity of $\mathrm{HoCO}_{2}$ measured below $5 \mathrm{~K}$ in various magnetic fields. Inset shows the nuclear specific heat coefficient $C_{N}$ [obtained from the fits of data for $0.8 \mathrm{~K} \leq T \leq 2 \mathrm{~K}$ to eqn (1)] as a function of magnetic field.

At zero magnetic field, the least squares fit of $C_{\mathrm{p}}$ gives nuclear specific heat coefficient $C_{\mathrm{N}}=2230 \pm 52 \mathrm{~mJ} \mathrm{~mol}^{-1} \mathrm{~K}^{-1}$, which is significantly higher than that of $\operatorname{PrAl}_{2} \cdot{ }^{55}$ Fig. 5 (inset) shows that $C_{\mathrm{N}}$ increases sharply from 0 to $20 \mathrm{kOe}$ and then tends to saturate in higher magnetic fields. $C_{\mathrm{N}}$ reaches $3370 \pm$ $66 \mathrm{~mJ} \mathrm{~mol} \mathrm{~K}^{-1}$ at $140 \mathrm{kOe}$. The tendency of $C_{\mathrm{N}}$ toward saturation when $H \geq 20 \mathrm{kOe}$ is similar to that observed in $\operatorname{PrAl}_{2},{ }^{55}$ and like in the latter this may be related to the fieldinduced change of crystal structure.

\section{X-ray powder diffraction}

It is well-established that $\mathrm{HoCo}_{2}$ compound undergoes a firstorder cubic-to-tetragonal transformation at $T_{\mathrm{C}}$, and then tetragonal-to-orthorhombic deformation at $T_{\mathrm{SR}}$, which appears to be the second-order transformation. ${ }^{7,8,11}$ This sequence of 
phase transitions was confirmed in our investigation and it agrees well with the magnetic and heat capacity measurements. The room temperature cubic lattice (space group $F d \overline{3} m$ ) transforms into the tetragonal one $\left(I 4_{1} /\right.$ amd $)$ at $T_{\mathrm{C}}$, which, in turn, becomes orthorhombic ( $F d d d$ ) below $T_{\mathrm{SR}}$.

In addition to the full-profile Rietveld refinement that was performed for all collected patterns and used for lattice parameters calculation, we monitored the lattice deformations of Laves phase structures using selected high-angle Bragg peaks. In case of the tetragonal deformation, any of the cubic $(00 l)$ reflections can be used. Fig. 6a shows that the (008) Bragg peak of the cubic structure splits into the (440) and (008) peaks when cooled below the $T_{\mathrm{C}}$. Since the Bragg angle of the more intense peak (440) is lower than the Bragg angle of (008) peak, and according to the Rietveld refinement, the $c$ lattice parameter contracts while the normalized to the non-distorted cubic structure values of the lattice parameters $a=b$ expand compared to the parent cubic structure. Therefore, our data confirm the results of ref. 7 and 47 , but disagree with ref. 8 and 11 , who reported the expansion of the $c$-axis during the tetragonal deformation.

As the sample is cooled below $T_{\mathrm{SR}}$, the (440) peak of the tetragonal phase further splits into two peaks of nearly even intensity indicating a distortion in the $a b$-plane, which results in the orthorhombic crystal structure of $\mathrm{HoCo}_{2}$ as its ground state. The known challenge here is to determine the symmetry of this crystal structure because two space groups, Fddd and Imma, provide nearly identical quality of fit during the refinement of the orthorhombic crystal structure. ${ }^{19}$ The notable difference between the two models is that the structure within the Fddd space group has a single Co position, while the Imma crystal structure has two symmetrically independent Co atoms. An early Mössbauer
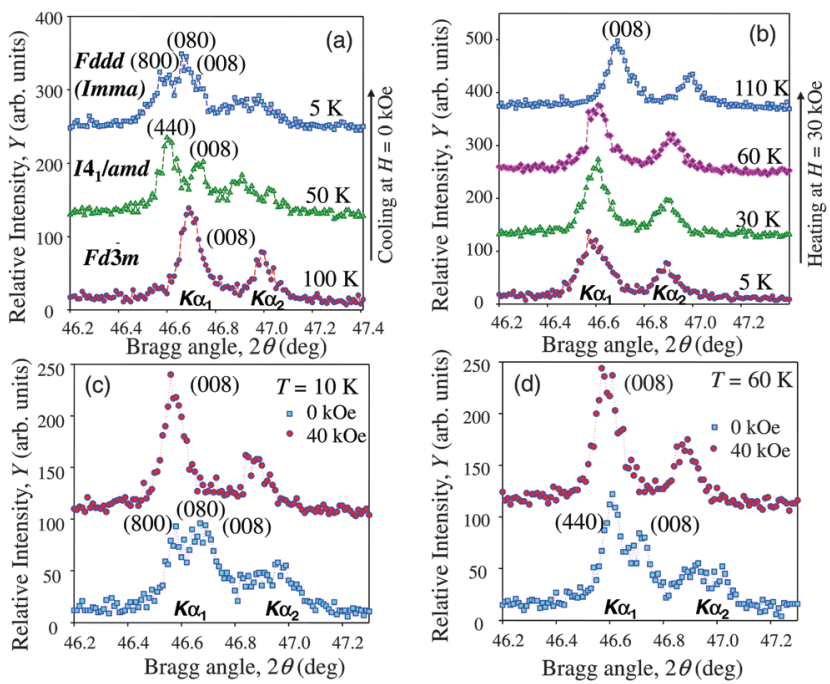

Fig. 6 Isofield evolution of the X-ray powder diffraction profile of the (008) Bragg peak measured as a function of temperature in zero (a) and $30 \mathrm{kOe}(\mathrm{b})$ magnetic fields. Restoration of the cubic (008) peak in the isothermal application of a $40 \mathrm{kOe}$ magnetic field at $10 \mathrm{~K}(\mathrm{c})$ and $60 \mathrm{~K}(\mathrm{~d})$ is shown as well. The data were collected using Mo $\mathrm{K} \alpha$ radiation (both the $\mathrm{K} \alpha 1$ and $\mathrm{K} \alpha 2$ contributions are shown). study indicated multiple Co sites in $\mathrm{HoCo}_{2}$ below $T_{\mathrm{SR}}{ }^{56}$ but the sample in that study was prepared with a significant amount of ${ }^{57} \mathrm{Fe}$ (its $T_{\mathrm{C}}$ was $90 \mathrm{~K}$ ) and it may not be used as a reliable reference point.

We performed first-principle calculations using both Fddd and Imma crystal structures (using crystallographic parameters determined at $10 \mathrm{~K}$ ), and it was found that the Fddd structure has lower total energy compared to the Imma structure. Therefore, the former should be considered as a stable ground state of $\mathrm{HoCo}_{2}$. Thus, in our work we will use the Fddd model to represent the orthorhombic crystal structure of $\mathrm{HoCo}_{2}$. However, at this point there is no overwhelming experimental evidence to choose one structure over the other and given the small energy difference between Fddd and Imma configurations it is possible that small changes in composition (such as the use of low-purity holmium metal in the synthesis of $\mathrm{HoCo}_{2}$ or the addition of $\mathrm{Fe}$ ) may lead to Imma structure being the stable crystal structure below $T_{\mathrm{SR}}$.

As seen in Fig. 1, the transition at $T_{\mathrm{C}}$ is sharp with no thermal hysteresis. However, because it is a first-order transition a phase separated state is expected to exist at the transition, even if over a narrow range of temperatures. Since there are no prior reports of phase co-existence in $\mathrm{RCo}_{2}$ compounds during transitions at $T_{\mathrm{C}}$, a detailed X-ray powder diffraction study of the temperature range from 76 to $83 \mathrm{~K}$ was performed. Fig. 7 shows that we were able to observe a co-existence of both high and low-temperature phases at $79.5 \mathrm{~K}$. This temperature is slightly different than the $T_{\mathrm{C}}=76 \mathrm{~K}$ value obtained from magnetic measurements, but such difference in transition temperature between bulk polycrystalline sample and a varnish-bonded powder sample is common, and was observed previously. ${ }^{49,57}$

The temperature dependence of the lattice parameters and the unit-cell volume is shown in Fig. 8 (determined with $5 \mathrm{~K}$ steps below $90 \mathrm{~K}$ ). A comparison of the zero-field temperature dependence with the 30 kOe data shows substantial changes in the degree of structural distortion. There is a sharp volume discontinuity at $T_{\mathrm{C}}$ at $H=0$ caused by a rapid expansion of the lattice constants in the $a b$ plane, while the $c$ lattice constant follows the normal thermal contraction trend of the cubic phase.

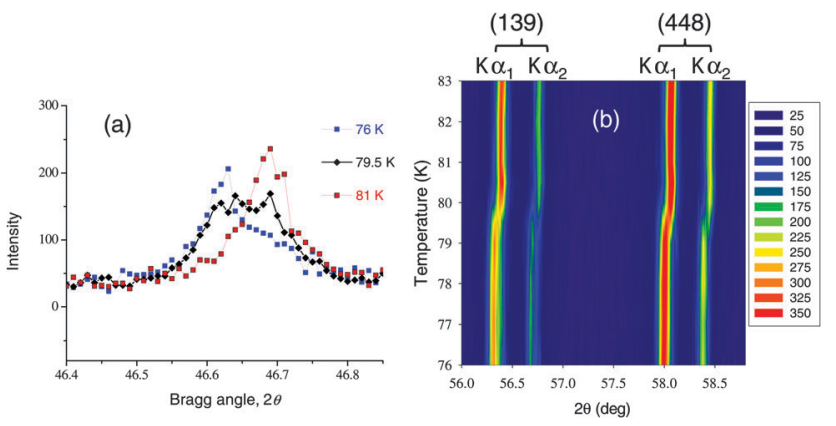

Fig. 7 (a) Temperature dependent profile of the (008) Bragg peak across the first order transition; the co-existence of Bragg reflections of both phases is seen at $79.5 \mathrm{~K}$ (only $\mathrm{K} \alpha 1$ component is shown). (b) Temperature dependent profile of the high-Bragg angle region of the $\mathrm{X}$-ray powder diffraction data of $\mathrm{HOCO}_{2}$ collected using $\mathrm{Mo} \mathrm{K} \alpha$ radiation. Phase separation is seen between 79 and $80 \mathrm{~K}$, especially in $\mathrm{K} \alpha 2$ reflections. 

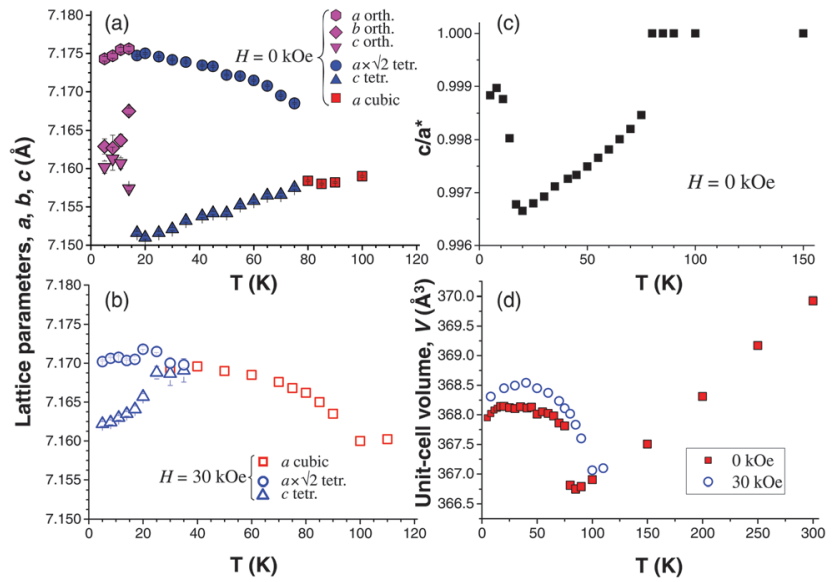

Fig. 8 The temperature dependence of the lattice parameters of $\mathrm{HoCO}_{2}$ measured in zero (a) and $30 \mathrm{kOe}(\mathrm{b})$ magnetic fields. The change of $\mathrm{c} / \mathrm{a}^{*}$ ratio with temperature is plotted for $\mathrm{H}=0 \mathrm{kOe}$ (c) and the temperature dependence of the unit cell volume is shown for both 0 and $30 \mathrm{kOe}$ applied magnetic fields (d). In the temperature dependence of $c / a^{*}$ ratio with temperature (c) the $a^{*}$ is defined as follows: for the cubic phase $a^{*}=a$, for the tetragonal phase $a^{*}=a \times \sqrt{ } 2$, and for the orthorhombic phase $a^{*}=\sqrt{ }(a \times b)$.

Essentially it means a rapid $c / a$ contraction as ferrimagnetic order sets in with EMA oriented along the $\langle 100\rangle$ direction. The change of the EMA to $\langle 110\rangle$ at $T_{\mathrm{SR}}$ brings, somewhat surprisingly, the value of the $c$ lattice parameter closer to the values of the in-plane parameters - in fact, the value of the $b$-parameter is closer to the $c$-, than to the $a$-parameter. The $c / a^{*}$ ratio for the orthorhombic phase, where $a^{*}=\sqrt{ }(a \times b)$, sharply increases at $T_{\mathrm{SR}}$ approaching unity. The change of lattice parameters at $T_{\mathrm{SR}}$ is rapid but not discontinuous. Neither the unit-cell volume (Fig. 8d) nor the entropy (calculated from the heat-capacity data, Fig. S5, ESI $\dagger$ ) show clear discontinuities at $T_{\mathrm{SR}}$, while both change discontinuously at $T_{\mathrm{C}}$, thus confirming the second-order type of the transition at $T_{\mathrm{SR}}$ and the first-order type of the phase transition at $T_{\mathrm{C}}$.

Application of magnetic field significantly affects crystallographic behaviour of $\mathrm{HoCO}_{2}$. It is noted that the analysis of field-dependent X-ray powder diffraction measurements of magnetically anisotropic materials has quantitative limitations due to the fact that the powder particles may respond to magnetic field differently depending on their orientation with respect to the vector of applied magnetic field. ${ }^{57}$ However, qualitative changes brought by magnetic field can be analysed and valuable information about structural response to applied magnetic field can be obtained.

For example, the extent of crystallographic distortion is suppressed when magnetic field of $H \geq 30 \mathrm{kOe}$ is applied to the system (compare Fig. 6a and b). The splitting of the (008) peak is no longer observed, although the peak shape is slightly different below and above the temperature of the structural transition. At lower temperatures $(<30 \mathrm{~K})$ the peak becomes broader and a better quality of Rietveld refinement fit is obtained when tetragonal model is used instead of cubic but the refinement of the orthorhombic model in $30 \mathrm{kOe}$ field becomes unstable.
This observation may explain why the magnetization step at $T_{\mathrm{SR}}$ becomes weaker in high magnetic fields. The suppression of structural distortion is further evidenced when the magnetic field is applied isothermally, for example at $10 \mathrm{~K}$ (Fig. 6c) and $60 \mathrm{~K}$ (Fig. 6d). In both cases a single (008) Bragg peak is observed in $40 \mathrm{kOe}$ field as opposed to the multiple peaks seen in a zero field.

The volume discontinuity at $T_{\mathrm{C}}$ extends over a large temperature range and is smaller overall in $H=30 \mathrm{kOe}$ compared to the one at $H=0$ (Fig. 8). This observation corroborates the high-field magnetization (Fig. 2b) and heat-capacity data, which show diminishing first-order character of the magnetic ordering transition with the increasing magnetic field strength. It is reasonable to expect that the volume discontinuity at $T_{\mathrm{C}}$ will completely disappear at a certain critical value of the applied magnetic field.

\section{Theoretical investigations}

The local spin density approximation including the onsite electroncorrelation $(\mathrm{LSDA}+U)^{58}$ approach has been employed to investigate the electronic structure, magnetic properties, and magnetostructural transformations of $\mathrm{HoCo}_{2}$. Calculations have been performed using the scalar relativistic version of the LSDA $+U$ method implemented in the tight binding linear muffin tin orbital (TB-LMTO) ${ }^{59}$ and full potential linear augmented plane wave (FP-LAPW) ${ }^{60}$ methods. The orbital dependent Coulomb and exchange interactions in LSDA $+U$ remove the degeneracy, and the $4 \mathrm{f}$ states split into different energy locations as prescribed by the site symmetry of Ho and Co atoms, and the number of partially filled orbitals in both spin channels obeying the Hund's spin and orbital rules in the Laves phase structures of $\mathrm{HoCo}_{2}$. Here, the spin orbit coupling of the $4 \mathrm{f}$ states $(\mathrm{J}=\mathrm{L}+\mathrm{S}$, for Ho atoms) follows Hund's rule. The calculations performed with different values of Hubbard $U$ ranging from $1 \mathrm{eV}$ to $7 \mathrm{eV}$ indicate that with the higher values of the $U$, the occupied $4 \mathrm{f}$ states are shifted to the lower energy while the unoccupied $4 \mathrm{f}$ states are shifted to the higher energy, as expected. The $k$-space integrations have been performed with $16 \times 16 \times 16$ Brillouin zone mesh, which was sufficient for the convergence of total energies, magnetic moments, and $4 \mathrm{f}$ and d splitting.

In the paramagnetic (PM) state, localized Ho-4f moments are randomly oriented and the net moment is zero. Considering that the experimentally observed effective magnetic moment (10.5 $\mu_{\mathrm{B}}$ per f.u., see Fig. S3, ESI $\dagger$ ) is close to the expected $g_{J}[J(J+1)]^{1 / 2}=10.61 \mu_{\mathrm{B}}$ of non-interacting $\mathrm{Ho}^{3+}$ ions and taking into account the itinerant character of Co $3 \mathrm{~d}$ electrons, magnetic moments of Co ions are nearly negligible in the PM state. At $T_{\mathrm{C}}$, Ho sublattice orders magnetically via indirect $4 \mathrm{f}-5 \mathrm{~d}-4 \mathrm{f}$ exchange interactions. As a result, the 5d PM DOS peak close to the Fermi level splits into the spin up and spin down DOS peaks (Fig. 9c) resulting in $\sim 0.3 \mathrm{eV}$ Ho-5d exchange splitting and $0.29 \mu_{\mathrm{B}}$ Ho-5d magnetic moment. Consequently, the Ho-5d/Co-3d hybridization in both spin up and spin down states promotes $4 \mathrm{f}-3 \mathrm{~d}$ exchange leading to antiparallel Ho-4f $\left(3.97 \mu_{\mathrm{B}}\right)$ and Co-3d $\left(-1.18 \mu_{\mathrm{B}}\right)$ spin moments and a substantial Co-3d exchange splitting $(\sim 1.15 \mathrm{eV})$ 

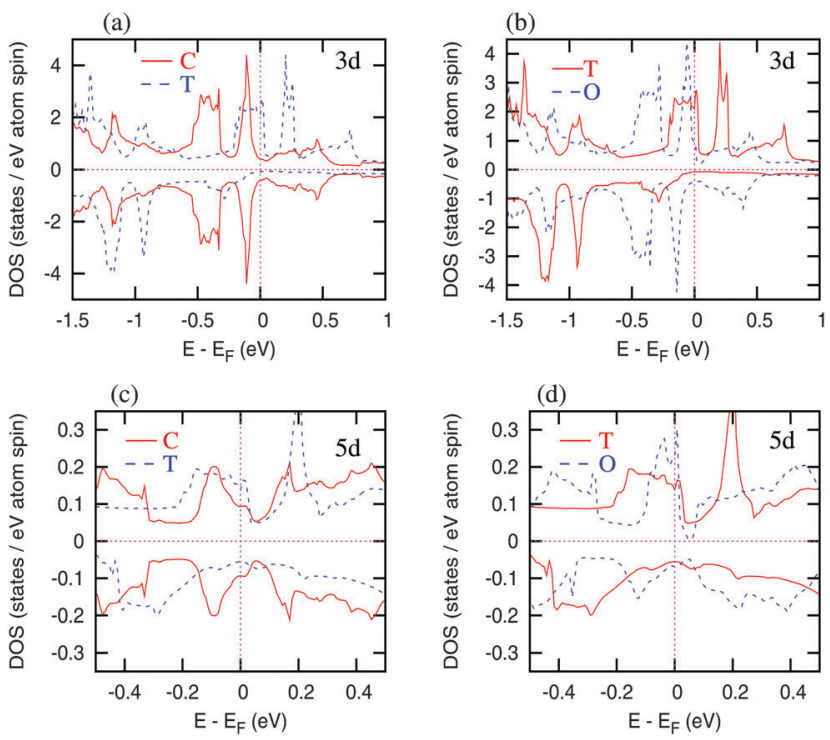

Fig. $93 d$ and $5 d$ DOS for the cubic (C), tetragonal (T), and orthorhombic $(\mathrm{O}-\mathrm{Fddd})$ structures of $\mathrm{HoCO}_{2}$.

(see Fig. 9a). This scenario is typical of an itinerant electron metamagnetic (IEM) transformation in which isotropic nonmagnetic PM state with cubic structure may abruptly distort into anisotropic ferrimagnetic (FIM) state with a tetragonal structure.

Total energy landscape (Fig. 10) indicates FIM orthorhombic Fddd structure (with $-0.07 \mu_{\mathrm{B}}$ Co magnetic moment) as the lowest energy (ground state) structure. The total energy of the FIM orthorhombic Imma structure (which has two split Co sites and $-0.07 / \mathrm{Co} \mu_{\mathrm{B}}$ on the Co atoms) is higher by $\sim 4.5 \mathrm{meV}$ per cell compared to the Fddd structure. Hence, the orthorhombic distortion in $\mathrm{HoCO}_{2}$ is not likely to be driven by the Co site splitting. The total energy of the FIM tetragonal structure is only slightly higher than that of both orthorhombic structures and substantially lower compared to $\mathrm{PM}$ cubic $\mathrm{HoCo}_{2}$. A substantial drop in the total energy during the PM cubic to the FIM tetragonal transformation and a minor reduction during the FIM tetragonal to FIM orthorhombic transformation are in line with experimentally observed discontinuous PM cubic to the FIM tetragonal and continuous FIM tetragonal to FIM orthorhombic transformations. Further, the exchange splitting of Co-3d states is weak in both orthorhombic (Fddd and Imma) structures leading to a small Co moment $\left(-0.07 \mu_{\mathrm{B}}\right.$ per Co) in contrast with a much larger Co moment $\left(-1.15 \mu_{\mathrm{B}}\right.$ per Co) obtained for the tetragonal structure. The diminishing Co moment is connected to the change of lattice parameters when the tetragonal structure transforms to the orthorhombic (Fig. 8). In the tetragonal structure the lattice parameter $a$ increases while the lattice parameter $c$ decreases $(c / a<1)$ following a sharp basal plane expansion at $T_{\mathrm{C}}$. At the tetragonal to orthorhombic transition (at $T_{\mathrm{SR}}$ ) the lattice parameters of the emergent orthorhombic structure change in such a way that the lattice distortion is reduced (the values of $c$ and $a$ and $b$ lattice parameters become closer to one another) essentially approaching the parent cubic structure as the temperature approaches zero.

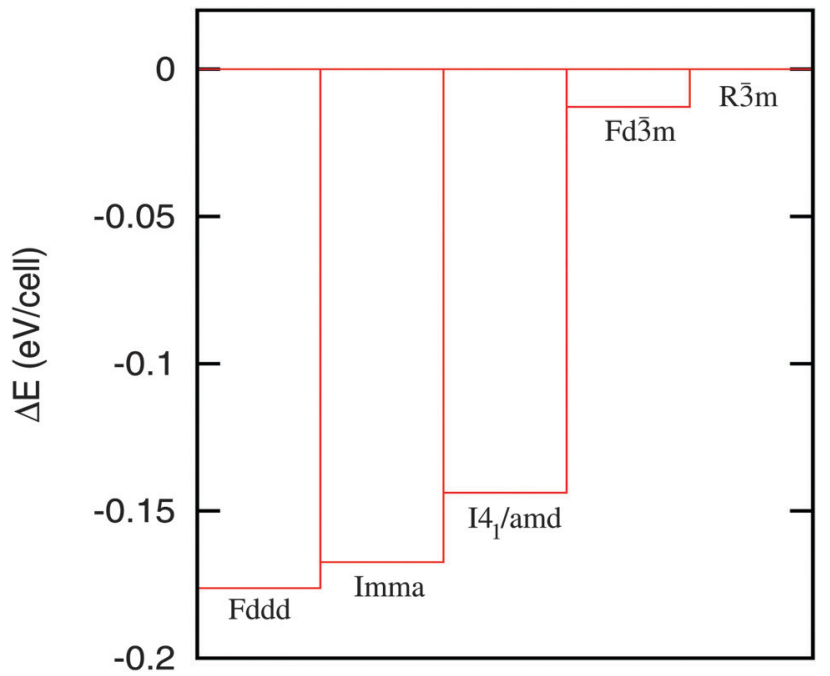

Fig. 10 Total energy landscape of possible structures of $\mathrm{HoCO}_{2}$. Fddd, Imma, $14_{1} / a m d, F d \overline{3} m$, and $R \overline{3} m$ are the respective space groups of the face-centered orthorhombic, body-centered orthorhombic, tetragonal, cubic, and hypothetical rhombohedral crystal structures.

The Hund's rule Ho-4f moment in both the orthorhombic and the tetragonal structures is $10 \mu_{\mathrm{B}}$ per Ho $\left(4 \mu_{\mathrm{B}}\right.$ per Ho contributed from $4 \mathrm{f}$ spins and $6 \mu_{\mathrm{B}}$ per Ho from $4 \mathrm{f}$ orbitals). The experimentally observed total moment is $8.4 \mu_{\mathrm{B}}$ per f.u. in the orthorhombic structure where the Co moment is nearly diminished. The decrease in Ho $4 \mathrm{f}$ moment is due to the $4 \mathrm{f}$ splitting (shown in Fig. 11), which is caused by the crystalline environment (tetrahedral arrangement) of Ho atoms in the C15 structure, and leads to the partial quenching of $4 \mathrm{f}$ orbital moment in this compound. Essentially, Ho moment is affected by the crystalline electric field splitting as has been shown before. ${ }^{17,18,20}$ The spin up $4 \mathrm{f}$ states split into three energy locations and the if spin down states, both occupied and unoccupied, each are split into two energy locations. These split states rigidly move towards the high energies in the orthorhombic structure compared to the tetragonal structure. Consequently, the spin up Co-3d DOS shifts to the lower energy and the spin down Co-3d DOS shifts to the higher energy (Fig. 9b) resulting in almost negligible Co-3d exchange splitting that gives rise to just $-0.07 \mu_{\mathrm{B}}$ Co moment in the orthorhombic $\mathrm{HoCO}_{2}$. The Ho-5d DOS features also get rearranged (Fig. 9d) and the spin polarized $5 \mathrm{~d}$ moment in the orthorhombic symmetry is reduced to $0.1 \mu_{\mathrm{B}}$ per Ho. This indicates that the $4 \mathrm{f}-4 \mathrm{f}$ and $4 \mathrm{f}-5 \mathrm{~d}$ interactions are weaker in the orthorhombic structure, leading to the reduced $4 \mathrm{f}-3 \mathrm{~d}$ interactions, and, consequently, to smaller Co moment below $T_{\mathrm{SR}}$. Since there is a reduction of Co moment in the orthorhombic structure, the total magnetic moment of $\mathrm{HoCo}_{2}$ with this structure is higher compared to the tetragonal structure (in which Ho and Co moments are antiparallel) as is observed experimentally (Fig. 2). The reduced but not extinct $5 \mathrm{~d}$ moment (from $4 \mathrm{f}-5 \mathrm{~d}$ interaction) still holds the Ho moments ferromagnetically aligned. Furthermore, with no significant Co magnetic moment, the IEM state (i.e., the FIM tetragonal structure) becomes unstable, and the system adopts a different 
(a)

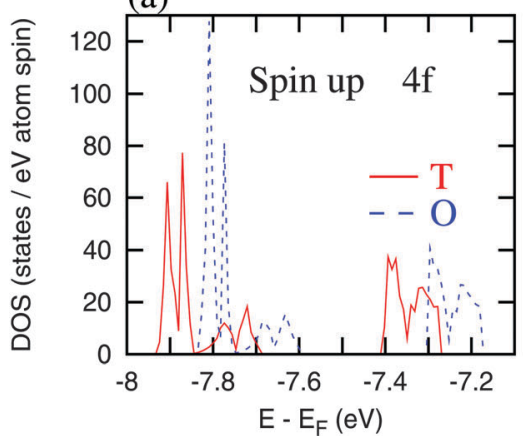

(b)

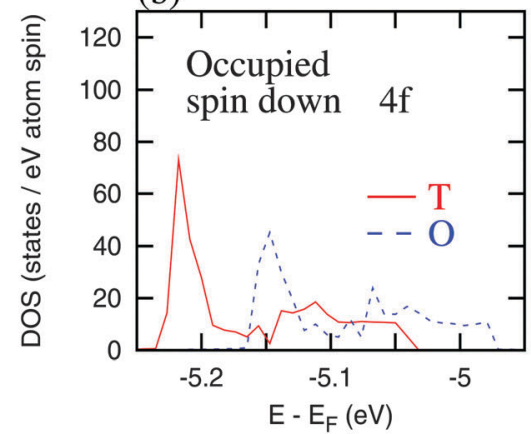

(c)

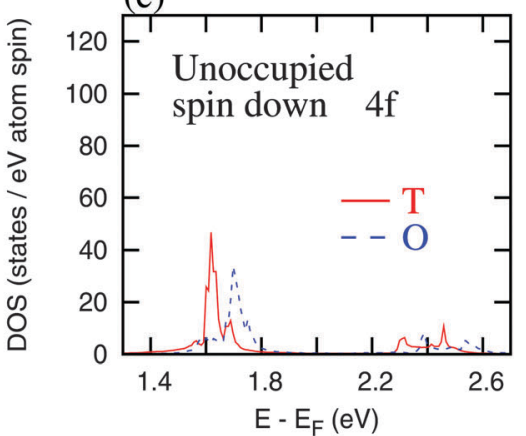

Fig. 11 The $4 \mathrm{f}$ density of states (DOS) for the tetragonal (T) and the orthorhombic (O - Fddd) structures of HoCO $2:$ (a) spin up $4 f \mathrm{DOS}$, (b) occupied spin down $4 \mathrm{f}$ DOS, and (c) unoccupied spin down $4 \mathrm{f}$ DOS.

structure (orthorhombic), where Ho becomes the primary source of magnetism.

Interestingly, a closely related Laves phase compound formed with non-magnetic aluminum instead of Co, i.e. $\mathrm{HoAl}_{2}$, has $\mathrm{FM}$ orthorhombic $(F d d d)$ ground state. $\mathrm{HoAl}_{2}$ exhibits a magnetic only transition (PM cubic to FM cubic) at $\sim 28 \mathrm{~K}$ and then undergoes a first order spin reorientation transformation during the orthorhombic $(F d d d)$ distortion below $\sim 20 \mathrm{~K}$. This structural anomaly is due to exchange splitting $(\sim 0.25 \mathrm{eV})$ opposed by the crystal field energy $(\sim 0.32 \mathrm{eV})$ of Ho- $5 \mathrm{~d} .{ }^{61}$ On the other hand, as noted above, $\mathrm{HoCo}_{2}$ practically loses Co magnetic moment in the orthorhombic ground state and the FIM (nearly FM) state here is due to the $4 \mathrm{f}-4 \mathrm{f}$ exchange mediated by the $4 \mathrm{f}-5 \mathrm{~d}$ interactions. The Ho-5d DOS in $\mathrm{HoCo}_{2}$ does not show crystal field splitting that was significant in $\mathrm{HoAl}_{2}$. Even though in the rare earth Laves phase compounds the underlying mechanisms for the low temperature magnetism and magnetostructural transformations may be different, they remain primarily driven by the magnetism of the $\mathrm{R}$ atoms.

\section{Conclusions}

Detailed experimental and first-principles study of $\mathrm{HoCo}_{2}$ explains the unusual low-temperature polymorphic transformation in terms of competition between the itinerant ferrimagnetic and the low-temperature state, where the Co magnetism is diminished, and both the magnetic and crystal structures are mainly defined by the magnetism of Ho. Three crystal structures, each associated with a distinctly different magnetic state of the compound, exist in three different temperature regions: (1) the high-temperature cubic structure, associated with the paramagnetic state, (2) the intermediate temperature tetragonal phase with the previously established IEM ferrimagnetic order, and (3) the low-temperature orthorhombic phase with the dominant ferromagnetism of Ho sub-lattice and the much reduced Co magnetism. First-principles calculations show that the ground state orthorhombic structure is expected to adopt the Fddd space group.

The title compound responds strongly to the varying external magnetic field, including suppression of the structural distortions both at $T_{\mathrm{C}}$ and $T_{\mathrm{SR}}$, limiting the stability region of the tetragonal $\mathrm{HoCo}_{2}$, changing the nature of the transition at $T_{\mathrm{C}}$ from first order to second, and the enhancement of the low-temperature Schottky anomaly, possibly, via the field-induced modification of low-temperature crystal structure. The rapid increase of $\mathrm{HoCo}_{2}$ heat capacity below $1 \mathrm{~K}$ makes it a promising passive regenerator material for the ultra-low temperature cryocooler applications.

\section{Acknowledgements}

The Ames Laboratory is operated for the U. S. Department of Energy by Iowa State University of Science and Technology under contract No. DE-AC02-07CH11358. This work was supported by the Department of Energy, Office of Basic Energy Sciences, Materials Sciences Division. The authors thank Mr Prathamesh Patil for taking SEM images.

\section{References}

1 M. Sagawa, et al., IEEE Trans. Magn., 1984, 20, 1584; J. F. Herbst, Rev. Mod. Phys., 1991, 63, 819; R. Skomski and J. M. D. Coey, Phys. Rev. B, 1993, 48, 15812.

2 K. H. J. Buschow, P. A. Naastepad and F. F. Westendorp, J. Appl. Phys., 1969, 40, 4029.

3 N. C. Koon, C. M. Williams and B. N. Das, J. Magn. Magn. Mater., 1991, 100, 173; A. E. Clark and H. S. Belson, Phys. Rev. $B, 1972, \mathbf{5}, 3642$.

4 O. Gutfliesch, M. A. Willard, E. Bruck, C. H. Chen, S. G. Shankar and J. P. Liu, Adv. Mater., 2011, 23, 821.

5 V. K. Pecharsky, K. A. Gschneidner, Jr., Y. Mudryk and D. Paudyal, J. Magn. Magn. Mater., 2009, 321, 3541.

6 F. H. Spedding and A. H. Daane, J. Metals, 1954, 6, 504.

7 E. Gratz, Solid State Commun., 1983, 48, 825.

8 A. S. Markosyan, Soviet Physics - Solid State, 1981, 23, 965.

9 K. A. Gschneidner, Jr. and V. K. Pecharsky, Z. Kristallogr., 2006, 221, 375.

10 V. V. Aleksandryan, R. Z. Levitin, A. S. Markosyan, V. V. Snegirev and A. D. Shchurova, Soviet Physics - JETP, 1987, 65, 502 .

11 R. Z. Levitin and A. S. Markosyan, J. Magn. Magn. Mater., 1990, 84, 247. 
12 E. Gratz, A. Lindbaum, A. S. Markosyan, H. Mueller and A. Yu. Sokolov, J. Phys.: Condens. Matter, 1994, 6, 6699.

13 S. Khmelevskyi and P. Mohn, J. Phys.: Condens. Matter, 2000, 12, 9453.

14 H. R. Kirchmayr and C. A. Poldy, J. Magn. Magn. Mater., 1978, 8, 1.

15 P. J. Brown, B. Ouladdiaf, R. Ballou, J. Deportes and A. S. Markosyan, J. Phys.: Condens. Matter, 1992, 4, 1103.

16 T. Goto, T. Sakakibara, K. Murata, H. Komatsu and K. Fukamichi, J. Magn. Magn. Mater., 1990, 90, 700.

17 A. Castets, D. Gignoux and B. Hennion, J. Magn. Magn. Mater., 1980, 15-18, 375.

18 E. W. Lee and P. Hendy, Physica, 1977, 86-88B, 163.

19 Y. G. Xiao, Q. Huang, Z. W. Ouyang, J. W. Lynn, J. K. Liang and G. H. Rao, J. Alloys Compd., 2006, 420, 29.

20 D. Gignoux, F. Givord and R. Lemaire, Phys. Rev. B, 1975, 12, 3878.

21 J. Voiron, A. Berton and J. Chaussy, Phys. Lett., 1974, 50A, 17. 22 G. Aubert, D. Gignoux, F. Givord, R. Lemaire and B. Michelutti, Solid State Commun., 1978, 25, 85.

23 V. Sechovsky, D. Vasylyev and J. Prokleska, Z. Naturforsch., 2007, 62b, 965.

24 T. D. Cuong, L. Havela, V. Sechovsky, A. V. Andreev, Z. Arnold, J. Kamarad and N. H. Duc, J. Alloys Compd., 1997, 262-263, 141.

25 C. M. Bonilla, J. Herrero-Albillos, A. I. Figueroa, C. CastanGuerrero, J. Bartolome, I. Calvo-Almazan, D. Schmitz, E. Weschke, L. M. Garcia and F. Bartolome, J. Phys.: Condens. Matter, 2014, 26, 156001.

26 R. M. Moon, W. C. Koehler and J. Farrell, J. Appl. Phys., 1965, 36, 978.

27 M. Rubinstein, P. Lubitz and N. C. Koon, J. Magn. Magn. Mater., 1981, 24, 288.

28 E. Gratz, R. Resel, A. T. Burkov, E. Bauer, A. S. Markosyan and A. Galatanu, J. Phys.: Condens. Matter, 1995, 7, 6687.

29 F. Garcia, L. C. Sampaio, A. Y. Takeuchi, H. Tolentino and A. Fontaine, J. Appl. Phys., 2000, 87, 5881.

30 D. Gignoux, F. Givord and W. C. Koehler, Physica, 1977, 86-88B, 165.

31 F. Bartolome, C. M. Bonilla, J. Herrero-Albillos, I. CalvoAlmazan, C. Castan, E. Weschke, D. Schmitz, D. Paudyal, Y. Mudryk, V. Pecharsky, K. A. Gschneidner, Jr., A. Stunault and L. M. Garcia, Eur. Phys. J. B, 2013, 86, 489.

32 J. Herrero-Albillos, F. Bartolome, L. M. Garcia, A. T. Young, T. Funk, J. Campo and G. J. Cuello, Phys. Rev. B, 2007, 76, 094409.

33 C. M. Bonilla, I. Calvo, J. Herrero-Albillos, A. I. Figueroa, C. Castan-Guerrero, J. Bartolome, J. A. Rodriguez-Velamazan, D. Schmitz, E. Weschke, D. Paudyal, V. K. Pecharsky, K. A. Gschneidner, Jr., F. Bartolome and L. M. Garcia, J. Appl. Phys., 2012, 111, 07E315.

34 C. M. Bonilla, D. Paudyal, J. Herrero-Albillos, V. K. Pecharsky, K. A. Gschneidner, Jr., L. M. Garcia and F. Bartolome, IEEE Trans. Magn., 2014, 50, 1700204.

35 W. Steiner, E. Gratz, H. Ortbauer and H. W. Camen, J. Phys. F: Met. Phys., 1978, 8, 1525.
36 O. Syshchenko, T. Fujita, V. Sechovsky, M. Divis and H. Fujii, J. Alloys Compd., 2001, 317-318, 438.

37 R. Hauser, E. Bauer and E. Gratz, Phys. Rev. B, 1998, 57, 2904.

38 O. Syshchenko, V. Sechovsky, M. Divis, T. Fujita, R. Hauser and H. Fujii, J. Appl. Phys., 2001, 89, 7323.

39 J. Herrero-Albillos, F. Bartolome, L. M. Garcia, F. Casanova, A. Labarta and X. Batlle, Phys. Rev. B, 2006, 73, 134410.

40 E. W. Lee and F. Pourarian, Phys. Status Solidi A, 1976, 33, 483.

41 N. V. Baranov and A. I. Kozlov, J. Alloys Compd., 1992, 190, 83.

42 S. A. Nikitin and A. M. Tishin, Cryogenics, 1991, 31, 166.

43 N. A. de Oliveira, J. Phys.: Condens. Mater, 2008, 20, 175209.

44 Materials Preparation Center, Ames Laboratory of US DOE, Ames, IA, USA, www.mpc.ameslab.gov.

45 V. K. Pecharsky, J. O. Moorman and K. A. Gschneidner, Jr., Rev. Sci. Instrum., 1997, 68, 4196.

46 A. P. Holm, V. K. Pecharsky, K. A. Gschneidner, Jr., R. Rink and M. N. Jirmanus, Rev. Sci. Instrum., 2004, 75, 1081.

47 E. Burzo, P. Vlaic, D. P. Kozlenko, S. E. Kichanov, N. T. Dang, A. V. Rutkauskas and B. N. Savenko, J. Alloys Compd., 2014, 584, 393.

48 E. M. Chudnovsky, W. M. Saslow and R. A. Serota, Phys. Rev. B, 1986, 33, 251.

49 R. Nirmala, Y. Mudryk, V. K. Pecharsky and K. A. Gschneidner, Jr., Phys. Rev. B, 2007, 76, 014407.

50 T. Saito and K. Hanashima, J. Magn. Magn. Mater., 2007, 310, 1540.

51 A. S. Chernyshov, Y. Mudryk, D. Paudyal, V. K. Pecharsky, K. A. Gschneidner, Jr., D. L. Schlagel and T. A. Lograsso, Phys. Rev. B, 2009, 80, 184416.

52 K. A. Gschneidner, Jr. and V. K. Pecharsky, Mater. Sci. Eng., 2000, A287, 301.

53 L. J. Sundström, in Handbook on the Physics and Chemistry of Rare Earths, ed. K. A. Gschneider, Jr. and L. Eyring, 1978, ch. 5, vol. 1, p. 379.

54 O. V. Lounasmaa, Phys. Rev., 1964, 133, A211; O. V. Lounasmaa, Phys. Rev., 1962, 128, 622; O. V. Lounasmaa, Phys. Rev., 1963, 129, 2460; O. V. Lounasmaa, Phys. Rev., 1962, 128, 1136.

55 A. K. Pathak, D. Paudyal, Y. Mudryk, K. A. Gschneidner, Jr. and V. K. Pecharsky, Phys. Rev. Lett., 2013, 110, 186405.

56 U. Atzmony, M. P. Dariel and G. Dublon, Phys. Rev. B, 1976, 14, 3713.

57 M. Zou, Y. Mudryk, V. K. Pecharsky, K. A. Gschneidner, Jr., D. L. Schlagel and T. A. Lograsso, Phys. Rev. B, 2007, 75, 024418.

58 V. I. Anisimov, F. Aryasetiawan and A. I. Lichtenstein, J. Phys.: Condens. Matter, 1997, 9, 767.

59 O. K. Andersen and O. Jepsen, Phys. Rev. Lett., 1984, 53, 2571.

60 P. Blaha, K. Schwarz, G. Madsen, D. Kvasnicka and J. Luitz, WIEN2k, An Augmented Plane Wave+Local Orbitals Program for Calculating Crystal Properties, Karlheinz Schwarz, Techn. Universität Wien, Austria, 2001, ISBN 3-9501031-1-2.

61 D. Paudyal, A. K. Pathak, V. K. Pecharsky and K. A. Gschneidner, Jr., J. Phys.: Condens. Matter, 2013, 25, 396002. 